\title{
Especies y sensibilidad a antifúngicos de cepas de Candida spp. aisladas de la cavidad bucal de pacientes con VIH de Paraguay
}

\author{
Belén Gayoso $^{1}$ (i) , Margarita Samudio ${ }^{1}$ (D) Carolina Duré $^{1}$ (i), Norma \\ Fariña $^{1}$ (D) Laura Alfonso $^{1}$ (D), Idalina Franco' ${ }^{1}$, Alicia Pereira ${ }^{2}$ (D), Desiré \\ Almirón ${ }^{3}$ iD , Aurelia Taboada ${ }^{3}$, Marcos Colmán 4 (iD) \\ ${ }^{1}$ Universidad Nacional de Asunción, Instituto de Investigaciones en Ciencias de la \\ Salud. San Lorenzo, Paraguay. \\ ${ }^{2}$ Laboratorio San Roque. Asunción, Paraguay. \\ ${ }^{3}$ Ministerio de Salud Pública y Bienestar Social, Instituto de Medicina Tropical, \\ PRONASIDA. Asunción, Paraguay. \\ ${ }^{4}$ Universidad Nacional de Asunción, Facultad de Ciencias Químicas. \\ San Lorenzo, Paraguay.
}

Cómo referenciar este artículo/ How to reference this article:
Gayoso B, Samudio M, Duré C, Fariña $N$, Alfonso $\boldsymbol{L}$, Franco $\boldsymbol{I}$, et al. Especies y sensibilidad a antifúngicos de cepas de Candida spp. aisladas de la cavidad bucal de pacientes con VIH de Paraguay. Mem. Inst. Investig. Cienc. Salud. 2021; 19(3): 1224

\section{RES U ME N}

Candida albicans es la levadura más aislada de la cavidad oral de personas con VIH. Sin embargo, el uso de antifúngicos ha incrementado el aislamiento de especies no $C$. albicans resistentes o con sensibilidad reducida. El objetivo del estudio fue determinar las especies de Candida y la sensibilidad a los antifúngicos de aislamientos de la cavidad bucal de pacientes con VIH, que acudieron al Instituto de Medicina Tropical entre julio a diciembre de 2019. Las levaduras se sembraron en Agar Cromogénico Candida $(C O N D A ®$, España) para la identificación presuntiva. Las colonias verdes se identificaron por PCR dúplex y las de coloración distinta o PCR negativa mediante el sistema comercial VITEK $® 2$. La susceptibilidad antifúngica de $C$. albicans se determinó por VITEK $® 2$, y las no albicans por el método de difusión de discos. De 278 levaduras, C. albicans se aisló en $70,9 \%$, seguido de C. tropicalis (8,3\%) y C. krusei (5\%). C. albicans presentó $93 \%$ de sensibilidad y $2 \%$ de sensibilidad dosis dependiente al fluconazol; $87 \%$ sensibles y $3 \%$ con sensibilidad intermedia frente a voriconazol; 97 , 98 y $100 \%$ sensibilidad a flucitosina, anfotericina B y equinocandinas, respectivamente. C. parapsilosis y $C$. tropicalis no registraron resistencias a fluconazol y voriconazol. Todos los aislamientos de C. glabrata fueron sensibles dosis dependientes a fluconazol y todos los aislamientos de $C$. krusei resultaron sensibles a voriconazol. La portación oral de Candida spp. resistentes a azoles podría llevar a fracasos terapéuticos en pacientes con VIH que desarrollen candidiasis orofaríngea.

Palabras clave: Candida, cavidad oral, VIH, antifúngicos. 


\title{
Species and sensitivity to antifungals of Candida spp. strains isolated from the oral cavity of patients with HIV from Paraguay
}

\begin{abstract}
A B S T R A C T
Candida albicans is the most isolated yeast from the oral cavity of people with HIV. However, the use of antifungals has increased the isolation of non-C. albicans resistant species or those with reduced sensitivity. The objective of the study was to determine the Candida species and the sensitivity to antifungals of isolates from the oral cavity of patients with HIV who attended the Institute of Tropical Medicine between July and December 2019. The yeasts were plated in Candida Chromogenic Agar (CONDA $®$, Spain) for presumptive identification. Green colonies were identified by duplex PCR and those with different staining or negative PCR using the commercial VITEK $® 2$ system. The antifungal susceptibility of $C$. albicans was determined by VITEK $® 2$, and non albicans by the disk diffusion method. Of 278 yeasts, C. albicans was isolated in $70.9 \%$, followed by C. tropicalis ( $8.3 \%$ ) and C. krusei (5\%). C. albicans presented $93 \%$ sensitivity and $2 \%$ dose-dependent sensitivity to fluconazole; $87 \%$ sensitive and $3 \%$ intermediate sensitivity to voriconazole; 97,98 , and $100 \%$ sensitive to flucytosine, amphotericin B, and echinocandins, respectively. C. parapsilosis and $C$. tropicalis did not register resistance to fluconazole and voriconazole. All C. glabrata isolates were dose-dependently sensitive to fluconazole and all $C$. krusei isolates were sensitive to voriconazole. The oral carriage of Candida spp. resistant to azoles may lead to therapeutic failures in HIV patients who develop oropharyngeal candidiasis.
\end{abstract}

Key words: Candida, oral cavity, HIV, antifungal.

\section{INTRODUCCIÓN}

La candidiasis orofaríngea es una de las infecciones oportunistas más frecuentes en los pacientes VIH positivos, con episodios recurrentes en aquellos con recuento de linfocitos $\mathrm{T} \mathrm{CD4}^{+}$inferior a $200 \mathrm{cel} / \mathrm{mL}$, por lo que es considerada un marcador pronóstico y diagnóstico del SIDA. Más del $95 \%$ de estos pacientes desarrollan al menos un episodio de candidiasis oral en la evolución de su enfermedad de base ${ }^{(1)}$.

Candida es una levadura que forma parte de la microbiota oral normal. Sin embargo, su portación, constituye un factor de riesgo para el desarrollo de candidiasis orofaríngea, sobre todo en los pacientes infectados por el VIH. C. albicans es el principal agente aislado, aunque en las últimas décadas ha disminuido su incidencia, siendo desplazado por especies diferentes como C. glabrata, C. krusei (actualmente denominada Pichia kudriavzevii), C. tropicalis, C. parapsilosis y C. dubliniensis ${ }^{(2-4)}$.

C. dubliniensis es una especie descrita por Sullivan et al. en $1995^{(5)}$. Su presencia es rara en la microbiota bucal normal, pero es detectado con frecuencia en la cavidad oral de pacientes con VIH. Presenta características fenotípicas y genotípicas similares a C. albicans con la que es confundida cuando se identifica mediante pruebas mínimas, ya que ambas tienen la capacidad de formar clamidosporas, tubos germinativos y cuando se utiliza el medio CHROMagar Candida las colonias que presentan son de color verde. Esto ha llevado a identificar incorrectamente la mayoría de los aislamientos, dificultando su análisis exhaustivo, por lo que para la identificación confirmatoria se recomienda utilizar técnicas moleculares ${ }^{(6)}$.

Las diversas especies de Candida varían en la susceptibilidad a los agentes antifúngicos. El fluconazol es uno de los más utilizados para la prevención y el tratamiento de la candidiasis oral, y se encuentra asociado a la aparición de cepas resistentes, como C. krusei, con resistencia intrínseca a los azoles o C. glabrata y $C$. dubliniensis, que desarrollan resistencia luego de la exposición, favoreciendo la prevalencia de los mismos en ciertas condiciones, representando una amenaza para la salud de los pacientes ${ }^{(7)}$. 
En Paraguay se ha publicado un artículo relacionado, en donde se identificaron especies de Candida de la cavidad oral de pacientes con HIV, mediante características bioquímicas y micromorfólogicas ${ }^{(8)}$. Aún no se han realizado estudios de portación en el país utilizando métodos moleculares que permitan diferenciar $C$. albicans de $C$. dubliniensis. Por lo que la finalidad del estudio fue determinar la prevalencia de las diversas especies de Candida que colonizan la cavidad oral de pacientes infectados por VIH, utilizando un método molecular y un método comercial. Además, determinar el patrón de susceptibilidad in vitro a los diferentes antifúngicos utilizados en el tratamiento y profilaxis de la candidiasis oral, para mejorar el manejo clínico y terapéutico de los mismos.

\section{MATERIALES Y MÉTODOS \\ Aislamientos}

Los aislamientos de Candida spp, eran provenientes de la cavidad bucal de pacientes con VIH/SIDA de diferentes edades y ambos sexos, hospitalizados y ambulatorios, con y sin lesión oral, con y sin tratamiento con antifúngicos, que acudieron al Instituto de Medicina Tropical, entre julio a diciembre de 2019. Fueron excluidos aislamientos no viables y/o aquellos con identificación incompleta.

\section{Procedimiento de trabajo}

Se analizaron 278 aislamientos de Candida spp, conservados en medio BHI (por sus siglas en inglés, Brain Heart Infusion), los cuales fueron sub cultivados para identificación presuntiva en Agar Cromogénico Candida (CONDA $®$, España), a $35{ }^{\circ} \mathrm{C}$ por 48 horas, en condiciones aeróbicas. Las colonias verdes fueron identificadas por el método molecular PCR dúplex, y las colonias con una coloración distinta a verde o PCR negativa, por el método comercial VITEK $^{\circledR} 2$. Se realizó la determinación de la susceptibilidad antifúngica a 155 aislamientos.

\section{Identificación molecular por PCR dúplex}

Las levaduras de coloración verde en el medio cromogénico fueron sub cultivadas en medio Sabouraud glucosado sin antibióticos e incubadas por 24 horas a $35^{\circ} \mathrm{C}$, y luego sometidas a una PCR dúplex estandarizada en el departamento de Microbiología del IICS/UNA, para diferenciar las especies de $C$. albicans y $C$. dubliniensis, mediante la amplificación del ADN ribosómico de la región espaciadora transcrita interna 1 y 2 (ITS-1 e ITS-2) (6).

Extracción del ADN: La extracción de ADN fue realizada utilizando el kit comercial Wizard $\AA$ Genomic DNA (Promega, USA), siguiendo las instrucciones del fabricante, con algunas modificaciones. En viales de $1,5 \mathrm{~mL}$ se dispensaron $300 \mu \mathrm{L}$ del buffer de lisis (Nuclei Lysis Solution), en los que se suspendieron una cantidad suficiente de colonias, luego se agregó una cucharadita de perlitas de vidrio de $0,5 \mathrm{~mm}$ y se sellaron con parafilm. Se agitaron los tubos a máxima velocidad por 1 minuto y se colocaron en baño de hielo 1 minuto (repitiendo estos dos pasos una vez más). Luego se centrifugaron a $13.000 \mathrm{rpm}$ durante 2 minutos, y los sobrenadantes transferidos a tubos nuevos de $1,5 \mathrm{~mL}$. Se incubaron los tubos a $80{ }^{\circ} \mathrm{C}$ por 5 minutos, y luego se procedió a enfriar a temperatura ambiente. Se agregó $1,5 \mu \mathrm{L}$ de RNAsa (RNase $A$ Solution) a cada tubo, y se mezcló invirtiendo 2 a 5 veces cada uno. Se incubaron a $37^{\circ} \mathrm{C}$ durante 30 minutos y luego se enfriaron a temperatura ambiente. Se agregó $100 \mu \mathrm{L}$ de solución de precipitación de proteínas (Protein Precipitation Solution) a cada tubo y se continuó siguiendo las instrucciones del fabricante. Por último, el ADN extraído se conservó a $-20^{\circ} \mathrm{C}$ hasta su uso.

Amplificación del ADN extraído: La amplificación específica de un fragmento génico de los aislamientos para la identificación de especie de $C$. albicans y $C$. dubliniensis se realizó empleando los cebadores "CALF-CALR" y "CDUF-CDUR", respectivamente (Tabla 1). 
Tabla 1: Secuencias de cebadores y tamaño de amplicones específicos utilizados para la amplificación de C. albicans y C. dubliniensis ${ }^{(6)}$.

\begin{tabular}{llll}
\hline $\begin{array}{l}\text { Gen } \\
\text { blanco }\end{array}$ & $\begin{array}{l}\text { Nombre de } \\
\text { Cebador }\end{array}$ & Secuencia $\left(\mathbf{5}^{\mathbf{\prime}^{\prime}} \mathbf{3}^{\mathbf{\prime}}\right)$ & $\begin{array}{l}\text { Tamaño del } \\
\mathbf{a m p l i c o ́ n ~} \mathbf{( p b})\end{array}$ \\
\hline ITS 2 & CALF & TGG TAA GGC GGG ATC GCT T & 100 \\
& CALR & GGT CAA AGT TTG AAG ATA TAC & \\
\hline ITS 1 & CDUF & AAA CTT GTC ACG AGA TTA TTT TT & 325 \\
ITS 2 & CDUR & AAA GTT TGA AGA ATA AAA TGG C & \\
\hline
\end{tabular}

Las condiciones de reacción utilizadas fueron las establecidas por Ahmad et al. (2012) (7), con algunas modificaciones. La amplificación se llevó a cabo con un volumen final de $15 \mu \mathrm{L}$ que contenía una mezcla con concentraciones finales de $1 \mathrm{x}$ de buffer, 1,5 mM de MgCl2, 0,1 mM de dNTPs, 0,5 U/tubo de Taq polimerasa, 0,2 $\mu \mathrm{M}$ de cada uno de los cebadores: CALF, CALR, CDUF y CDUR, $2 \mu L$ de ADN y agua estéril. Para la amplificación se utilizó el termociclador SimpliAmp ${ }^{\text {TM }}$ Thermal Cycler (Thermo Físher Scientific, USA), en donde las condiciones del ciclo incluyeron una desnaturalización inicial a $95{ }^{\circ} \mathrm{C}$ durante 5 minutos, seguida de 30 ciclos a $95{ }^{\circ} \mathrm{C}$ durante 1 minuto, $55^{\circ} \mathrm{C}$ durante 30 segundos y $72{ }^{\circ} \mathrm{C}$ durante 1 minuto y una extensión final a $72{ }^{\circ} \mathrm{C}$ durante 10 minutos. En cada tanda de amplificación se incluyeron ADN de C. albicans ATCC 90028 y una cepa conocida de C. dubliniensis como controles positivos. Como control negativo se utilizó la mezcla de reacción sin el agregado de ADN.

Detección del producto de amplificación: Los productos amplificados fueron detectados mediante electroforesis en gel de agarosa al $2 \%$ (100V, $30 \mathrm{~min}$ ), utilizando SYBR $®$ Safe DNA gel stain (Invitrogen, USA) para la visualización del ADN, donde los tamaños de los fragmentos para C. albicans y C. dubliniensis fueron de 100 y $325 \mathrm{pb}$ respectivamente, visualizados con el transiluminador de rayos UV Digimage System GDS-01 (mrc, Israel).

\section{Identificación fenotípica por VITEK ${ }^{\circledR} 2$ (BioMérieux)}

Los aislamientos que presentaron colonias verdes en medio cromogénico no identificados por la PCR dúplex, como también aquellos aislamientos que presentaron otros colores fueron identificados mediante el sistema automatizado VITEK ${ }^{\circledR} 2$ (BioMérieux, Francia). Cada aislado fue sub-cultivado en agar Sabouraud glucosado e incubado 24 horas a $35^{\circ} \mathrm{C}$. El inóculo fue preparado a partir de la suspensión de levaduras en $3 \mathrm{~mL}$ de solución fisiológica - agua destilada (1:1), hasta una turbidez equivalente a 1,8-2,2 de McFarland. El inóculo final se dispensó en la tarjeta YST ID a través de un tubo de poliestireno estéril, y se incubó en el equipo VITEK ${ }^{\circledR} 2$. Para la identificación de especies el sistema utiliza el perfil de asimilación de carbohidratos y ácidos orgánicos y la interpretación es realizada según la base de datos del equipo.

\section{Perfil de susceptibilidad antifúngica}

Se realizó el perfil de susceptibilidad antifúngica a los primeros 100 aislamientos de $C$. albicans por el sistema comercial VITEK ${ }^{\circledR} 2$, y a 55 aislamientos de $C$. no albicans por el método de difusión en agar, utilizando los puntos de corte propuestos por el Comité Internacional de Estándares de Laboratorio Clínico (CLSI) $(9,10)$. Como control se utilizaron cepas de referencia C. parapsilosis ATCC 22019 y C. krusei ATCC 6258.

Método de difusión en agar con discos de antifúngicos: Se realizó el antifungigrama por este método a 23 cepas de C. tropicalis, 11 de C. parapsilosis, 14 de C. krusei, y 7 de C. glabrata. Fueron utilizados discos de fluconazol de $25 \mu \mathrm{g}$ y voriconazol de $1 \mu \mathrm{g}$. Los diámetros de inhibición fueron interpretados como sensible, intermedio, sensible dosis-dependiente o resistente, empleando los puntos de corte recomendados por el CLSI para Candida spp $(9,10)$.

Método automatizado - VITEK ${ }^{\circledR}$ 2: Se ensayaron por este método 100 levaduras de C. albicans, que fueron reaisladas del medio cromogénico en agar Sabouraud glucosado e incubadas 24 horas a $35{ }^{\circ} \mathrm{C}$. Fueron utilizadas tarjetas del sistema VITEK ${ }^{\circledR} 2$ AST-YSO7, que contienen diluciones seriadas de los siguientes antifúngicos: 
anfotericina B $(0,25$ a $16 \mu \mathrm{g} / \mathrm{mL})$, caspofungina (1 a $8 \mu \mathrm{g} / \mathrm{mL})$, fluconazol (1 a 64 $\mu \mathrm{g} / \mathrm{mL}$ ), flucitosina (4 a $64 \mu \mathrm{g} / \mathrm{mL})$, micafungina $(0,06$ a $4 \mu \mathrm{g} / \mathrm{mL}$ ) y voriconazol $(0,12$ a $8 \mu \mathrm{g} / \mathrm{mL}$ ). Se procedió siguiendo estrictamente las instrucciones establecidas por el fabricante.

Se asignó una interpretación de categoría sensible, intermedio, dosis-dependiente o resistente de acuerdo a la concentración inhibitoria mínima (CIM), según los puntos de corte definidos en el documento del CLSI (9). El CLSI no ha establecido puntos de corte para anfotericina $\mathrm{B}$, ni para flucitosina. Por lo tanto, anfotericina B fue considerada como sensible a una CIM $\leq 1 \mu \mathrm{g} / \mathrm{mL}$ y flucitosina a una CIM $\leq 4 \mu \mathrm{g} / \mathrm{mL}$, de acuerdo a la interpretación emitida por el sistema VITEK ${ }^{\circledR} 2$ (Basados en criterios cinéticos y poblacionales).

\section{Gestión y análisis de los datos}

Los datos fueron consignados en una hoja de cálculo de Microsoft Excel, y los resultados fueron expresados en forma de distribución de frecuencias.

\section{Consideraciones éticas}

El proyecto cuenta con la aprobación del Comité de Ética de la Facultad de Ciencias Químicas - UNA, según el código CEI 568/19. El presente trabajo, se ajustó a los principios éticos de la investigación clínica: Respeto, Beneficencia y Justicia. Los datos clínicos, demográficos y los resultados de los pacientes, fueron mantenidos en forma confidencial utilizando códigos de identificación garantizando la privacidad de los mismos.

\section{RESULTADOS \\ Muestra}

Se estudiaron 278 aislamientos de Candida provenientes de la cavidad oral de 242 pacientes con VIH, de los cuales, 209 presentaron aislamientos únicos y 33 mixtos; de estos últimos se obtuvieron 69 aislamientos.

\section{Características socio-demográficas y clínicas de los pacientes con VIH/SIDA}

El $62 \%$ de los 242 pacientes eran hombres, el resto mujeres; el rango de edad fue de 1 a 75 años con una media de $39( \pm 11)$ años. De 232 pacientes, el rango de edad con mayor cantidad de aislamientos fue de 31 a 40 años (34,5\%). De 216 pacientes, $77,1 \%$ provenían del Departamento Central y Asunción, el resto del interior del país. El $71 \%$ de los pacientes eran ambulatorios y el $29 \%$ se encontraban hospitalizados. Las especies más aisladas en ambos grupos fueron $C$. albicans, $C$. tropicalis y $C$. krusei (datos no mostrados).

De 202 pacientes con aislamientos, el 43,6\% presentaba un recuento de linfocitos TCD4+ inferior a $200 \mathrm{cel} / \mathrm{mL}$ y $35,1 \%$ de 200 - $500 \mathrm{cel} / \mathrm{mL}$ TCD4+. De 232 pacientes, el $19 \%$ presentó candidiasis pseudomembranosa y el 5,6\% candidiasis eritematosa. De 221 pacientes, 11,3 \% recibieron alguna vez tratamiento profiláctico con fluconazol.

Considerando solo los pacientes con aislamientos únicos, no hubo relación estadísticamente significativa entre los aislamientos de $C$. albicans y $C$. no albicans con respecto al sexo, la edad, el recuento de linfocitos $T$ CD4+, presencia de lesión, tratamiento previo, tratamiento instaurado y el tipo de paciente $(p>0,05)$ (datos no mostrados).

\section{Identificación de las cepas de Candida spp.}

De los 278 aislamientos de Candida estudiados, 225 desarrollaron variadas tonalidades de color verde, y los 53 restantes presentaron otras coloraciones (rosa claro, rosa obscuro, blanco, purpura). Los 225 aislamientos de color verde se sometieron a una PCR dúplex (previamente estandarizada para la identificación de $C$ albicans-C dubliniensis), de los cuales 197 resultaron positivos para $C$. albicans, 1 positivo para $C$. dubliniensis y los 27 restantes dieron negativos tanto para $C$ albicans y $C$ dubliniensis. Estos aislamientos negativos por el método molecular más las 53 que habían desarrollado una coloración distinta a verde se identificaron por el sistema comercial VITEK ${ }^{\circledR} 2$, analizándose en total 80 cepas por este método. 


\section{Frecuencia de cepas de Candida spp. aisladas de la cavidad oral de pacientes con VIH}

La especie más frecuente en la cavidad oral de pacientes con VIH fue $C$. albicans (70,9\%), seguida de C. tropicalis ( $8,3 \%)$ y C. krusei (5\%). En menor proporción fueron identificadas C. parapsilosis, C. lusitaniae, C. glabrata, C. famata, C. kefyr, C. zeylanoides, C. dubliniensis, C. colliculosa, C. intermedia, C. lipolytica, C. pelliculosa y C. spherica. En total 29,1\% $(n=81)$ correspondió a especies de C. no albicans. (Tabla 2).

Tabla 2: Frecuencia de especies de Candida spp. identificadas por PCR dúplex y VITEK $^{\circledR} 2$, aisladas de la cavidad oral de pacientes con VIH $(n=278)$.

\begin{tabular}{|c|c|c|}
\hline Especies de Candida & $\mathbf{n}$ & $\%$ \\
\hline C. albicans & 197 & 70,9 \\
\hline C. tropicalis & 23 & 8,3 \\
\hline C. krusei & 14 & 5 \\
\hline C. parapsilosis & 11 & 4 \\
\hline C. Iusitaniae & 9 & 3,2 \\
\hline C. glabrata & 7 & 2,5 \\
\hline C. famata & 4 & 1,4 \\
\hline C. kefyr & 4 & 1,4 \\
\hline C. zeylanoides & 3 & 1,1 \\
\hline C. dubliniensis & 1 & 0,4 \\
\hline C. colliculosa & 1 & 0,4 \\
\hline C. intermedia & 1 & 0,4 \\
\hline C. lipolytica & 1 & 0,4 \\
\hline C. pelliculosa & 1 & 0,4 \\
\hline C. spherica & 1 & 0,4 \\
\hline Total & 278 & 100 \\
\hline
\end{tabular}

De los 278 aislamientos, 69 (24,8 \%) provenían de 33 aislamientos mixtos de Candida spp. (Tabla 3 ). Las asociaciones más comunes fueron $C$. albicans con $C$. tropicalis $(n=7)$, seguida de C. albicans $+C$. krusei $(n=4)$. Las especies no albicans también mostraron asociación entre ellas, pero en menor cantidad.

Tabla 3: Número de aislamientos mixtos de Candida spp. en la cavidad oral de pacientes con VIH $(n=33)$.

\begin{tabular}{lc}
\hline $\begin{array}{l}\text { Especies } \\
\text { combinadas }\end{array}$ & Candida \\
Aislamientos mixtos (n) \\
\hline C. albicans + C. tropicalis & 7 \\
C. albicans + C. krusei & 4 \\
C. albicans + C. lusitaniae & 3 \\
C. albicans + C. famata & 3 \\
C. albicans + C. kefyr & 2 \\
C. albicans + C. glabrata & 2 \\
C. albicans + C. parapsilosis & 2 \\
C. albicans + C. pelliculosa & 1 \\
C. albicans + C. lipolytica & 1 \\
C. albicans + C. tropicalis + C. & 1 \\
glabrata & \\
C. albicans + C. krusei + C. 1 \\
\hline
\end{tabular}




\begin{tabular}{lc}
\hline parapsilosis \\
C. albicans + C. krusei + C. \\
glabrata & 2 \\
C. tropicalis + C. krusei & 1 \\
C. tropicalis + C. lusitaniae & 1 \\
C. tropicalis + C. parapsilosis & 1 \\
C. glabrata + C. intermedia & 33 \\
Total
\end{tabular}

\section{Perfil de sensibilidad antifúngica}

De los 100 aislamientos de $C$. albicans analizados, el $93 \%$ fue sensible a fluconazol y el $2 \%$ sensible dosis dependiente, $87 \%$ sensible a voriconazol y $3 \%$ con sensibilidad intermedia. A caspofungina y micafungina resultaron sensibles el $100 \%$, a anfotericina B $98 \%$ y a flucitosina $97 \%$ de los aislamientos (Tabla 4).

Tabla 4: Susceptibilidad antifúngica de C. albicans $(n=100)$.

\begin{tabular}{llllllllllllll}
\hline & FLU* & & & \multicolumn{1}{l}{ VRC* } & CAS* & MCF* & AB** & \multicolumn{1}{c}{ FCT** } \\
\hline S & SDD & $\mathbf{R}$ & S & I & $\mathbf{R}$ & S & S & S & $\mathbf{R}$ & $\mathbf{S}$ & $\mathbf{R}$ \\
93 & 2 & 5 & 87 & 3 & 10 & 100 & 100 & 98 & 2 & 97 & 3 \\
\hline
\end{tabular}

C. tropicalis y C parapsilosis mostraron $100 \%$ de sensibilidad frente a fluconazol y a voriconazol. Los 7 aislamientos de C. glabrata fueron sensibles dosis dependiente frente a fluconazol; esta especie no posee categoría sensible frente a fluconazol y tampoco posee puntos de corte clínico frente a voriconazol. C. krusei es considerado intrínsecamente resistente a fluconazol. (Tabla 5).

Tabla 5: Susceptibilidad antifúngica de cepas de $C$. no albicans.

\begin{tabular}{|c|c|c|c|c|c|}
\hline \multirow{3}{*}{$\begin{array}{l}\text { Especies de } \\
\text { Candida }\end{array}$} & \multirow[b]{3}{*}{ n (\%) } & \multicolumn{4}{|c|}{ Susceptibilidad antifúngica - n (\%) } \\
\hline & & \multicolumn{3}{|c|}{ FLU $(n=55)$} & \multirow{2}{*}{$\begin{array}{l}\begin{array}{l}\text { VRC } \\
(n=48)\end{array} \\
S\end{array}$} \\
\hline & & $\mathbf{S}$ & SDD & $\mathbf{R}$ & \\
\hline C. tropicalis & $23(41,8)$ & $23(100)$ & $0(0)$ & $0(0)$ & $23(100)$ \\
\hline C. parapsilosis & $11(20)$ & $11(100)$ & $0(0)$ & $0(0)$ & $11(100)$ \\
\hline C. glabrata & $7(12,7)$ & - & $7(100)$ & $0(0)$ & - \\
\hline C. krusei* & $14(25,5)$ & - & - & $14(100)$ & $14(100)$ \\
\hline Total & $55(100)$ & $34(62)$ & $7(13)$ & $14(25)$ & $48(100)$ \\
\hline
\end{tabular}

(-) Puntos de corte no proporcionados por el CLSI M60.

*C. krusei es considerado intrínsecamente resistente al fluconazol.

FLU = Fluconazol. VRC= Voriconazol. $\mathrm{S}=$ Sensible. $\mathrm{SDD}=$ Sensible dosis-dependiente.

\section{DISCUSIÓN}

La especie de Candida aislada con más frecuencia de la cavidad oral de pacientes con VIH en este estudio fue $C$. albicans, que en el medio cromogénico de cribado desarrolló una coloración verde, esta interpretación es subjetiva y teniendo en cuenta que, se puede obtener varias tonalidades de este color en este medio y que no discriminan complejo de especies, es necesario un método más específico para la confirmación de especie, como fue el uso de la PCR dúplex que además de ser sensible y específico, posee la ventaja de diferenciar en una sola reacción dos especies, C. dubliniensis y $C$. albicans, que comparten características fenotípicas y pertenecen al mismo complejo de especies. 
La colonización oral por especies de Candida es un evento frecuente en pacientes VIH positivos. Esto aumenta el riesgo de desarrollar candidiasis orofaríngea en los mismos $^{(11)}$. Varios estudios han mostrado que $C$. albicans es la especie aislada con mayor frecuencia en estos pacientes ${ }^{(12-15)}$. En este estudio se obtuvo similar frecuencia a lo reportado por Clark et al., con $71,8 \%^{(16)}$. Otros autores mencionan prevalencias entre el $45 \%$ y $80 \%{ }^{(4,14,15)}$. Su presencia como colonizador y patógeno oportunista se debe probablemente a la capacidad que tiene $C$. albicans para adherirse a las células del epitelio bucal ${ }^{(17)}$.

La mayoría de los aislamientos $C$. dubliniensis no se han identificado correctamente por métodos convencionales, debido la estrecha relación fenotípica y genotípica con $C$. $a_{\text {albicans }}{ }^{(18)}$. La presencia de esta especie no era conocida en el país, ya que la misma pasaba inadvertida, probablemente por falta de una correcta identificación. La PCR dúplex aplicada en este estudio ha permitido diferenciar por primera vez una cepa de C. dubliniensis de 197 cepas de C. albicans en Paraguay, con una frecuencia de 0,4\%, muy por debajo de los otros estudios como los realizados por Castro et al. en Colombia, y Luque et al. en Argentina, que muestran una frecuencia elevada de C. dubliniensis de $8,9 \%$ y $20,2 \%$, respectivamente ${ }^{(15,19)}$. Esta especie posee prevalencias variables según la región geográfica, reportándose 4,6 \% en Turquía ${ }^{(20)}$, $7,5 \%$ en Nigeria ${ }^{(4)}, 5,4 \%$ en Brasil ${ }^{(21)}$ y $6 \%$ en México ${ }^{(22)}$.

Este hallazgo coincide con todos los estudios epidemiológicos que han demostrado la asociación de esta especie con la portación oral en pacientes con VIH/SIDA. Sin embargo, también se han indicado aislamientos en individuos con otras patologías como la diabetes, y ha estado ausente o presente en niveles muy bajos en individuos sanos hasta la fecha ${ }^{(23,24)}$. Los métodos moleculares son generalmente más rápidos, sensibles, específicos y confiables comparados a los métodos fenotípicos ${ }^{(5)}$. Debido a exigencias de costo, infraestructura y experiencia técnica requerida, no son factibles en el laboratorio como método de rutina. Además, la diferenciación entre $C$. albicans, la especie más virulenta, del sustituto menos virulento, $C$. dubliniensis es clínicamente ventajoso ${ }^{(22)}$.

Aunque $C$. albicans continúa siendo la especie dominante en la cavidad oral de pacientes infectados por VIH, las especies de $C$. no albicans están aumentando ${ }^{(4,25)}$. En este estudio se obtuvo un porcentaje del 29,1\% para estas especies, semejante a lo publicado por Clark et al. en México ${ }^{(16)}$. Otros autores han encontrado prevalencias entre $20 \%$ y $55 \%^{(4,14,15)}$. El sistema automatizado VITEK ${ }^{\circledR} 2$ es un método confiable, simple y efectivo para la identificación de las especies del género Candida clínicamente relevantes, que permitió la identificación de levaduras no $C$. albicans, no $C$. dubliniensis. La desventaja es su costo elevado, además que con cierta frecuencia se requieren de pruebas adicionales para la identificación de especies con perfiles bioquímicos similares ${ }^{(26,27)}$.

Entre las especies de $C$. no albicans se identificaron $C$. tropicalis y $C$. krusei, en mayor proporción $(8,3 \%$ y $5 \%$, respectivamente), coincidiendo en las especies predominantes con Garzón et al., quienes obtuvieron 18,8 \% de C. tropicalis y $12,5 \%$ de C. rrusei $^{(28)}$. La identificación de Candida a nivel de especie es importante ya que algunas cepas poseen resistencia intrínseca a los agentes antifúngicos empleados habitualmente ${ }^{(29)}$. Nweze et al. en Nigeria, encontraron una prevalencia elevada de C. tropicalis con $18,3 \%$ y C. parapsilosis con $15 \%{ }^{(4)}$. Los estudios realizados por Castro et al., y Luque et al., muestran una frecuencia elevada de C. glabrata de 7,9\% y $5,6 \%$, respectivamente ${ }^{(15,19)}$.

En el estudio realizado por Sanabria et al. (2006) en Paraguay, se obtuvo una frecuencia predominante de C. albicans (58\%), seguido de C. tropicalis (23\%) y C. krusei $(16 \%)^{(8)}$, demostrando que se mantiene la población de especies prevalentes en el país. Sin embargo, en dicho estudio la frecuencia de especies no albicans fue superior debido probablemente a que todos los pacientes con VIH presentaban candidiasis orofaríngea. En cambio, en este estudio, más del $70 \%$ de los pacientes no presentaban lesión oral.

La asociación de especies más frecuente en este estudio fueron $C$. albicans con $C$. tropicalis seguido de C. albicans con C. krusei. Luque et al. y Clark et al., indican una combinación prevalente de $C$. albicans con $C$. glabrata $^{(16,19)}$, mientras que Gutiérrez et 
al., informan C. albicans con C. krusei ${ }^{(30)}$. La coexistencia de varias especies de Candida en la cavidad oral ha sido señalado por varios investigadores $(12,16,19)$. Esto aumenta el riesgo de desarrollar candidiasis orofaríngea en individuos con VIH, sobre todo si la colonización es por levaduras con resistencia al fluconazol como $C$. krusei y C. glabrata ${ }^{(31)}$.

En este estudio, la sensibilidad se determinó por dos metodologías diferentes (por Vitek $^{\circledR} 2$ y por difusión con discos), debido factores económicos y a la escasa disponibilidad de las tarjetas se realizó la sensibilidad de los aislamientos de $C$. albicans por Vitek ${ }^{\circledR} 2$ y de las otras especies de Candida que presentan puntos de corte en el manual del CLSI por difusión con discos.

Con relación a la sensibilidad a los azoles, $C$. albicans presentó buena sensibilidad al fluconazol, mayor al $90 \%$. Resultados muy variables se han reportado en diversos estudios realizados en poblaciones similares. Tercas et al. encontraron $100 \%$ de sensibilidad al fluconazol ${ }^{(25)}$, Brito et al. reportaron $67 \%$ de sensibilidad y $33 \%$ de sensibilidad dosis dependiente (SDD), si bien en un pequeño número de aislamientos (32); Khedri et al. hallaron mayor resistencia, 57,4\% de aislamientos sensibles, $13 \%$ sensibles dosis dependientes y $29,6 \%$ resistentes $^{(33)}$.

Se encontró similar sensibilidad de $C$ albicans frente a voriconazol a lo reportado por Khedri et al. que informaron $83,3 \%$ de aislamientos sensibles de C. albicans, 7,4 $\%$ SDD y $9,3 \%$ resistentes, en candidiasis orofaríngea. Otros autores hallaron $100 \%$ de sensibilidad de $C$. albicans frente a voriconazol ${ }^{(16,24,33)}$. Con relación al trabajo publicado recientemente en nuestro país, por Aguilar et al. en aislamientos provenientes de hemocultivos, la resistencia al voriconazol que encontramos es elevada ${ }^{(34)}$. Probablemente la mayor resistencia es debida a la utilización frecuente de azoles en la población estudiada, tanto para profilaxis como para el tratamiento. Además, se debe considerar que la sensibilidad no fue realizada por el método de referencia, si bien se ha reportado buena correlación con la metodología utilizada ${ }^{(35)}$. Cabe resaltar que el origen de los aislamientos no es el mismo. Esto constituye una alarma, pues la portación de estas levaduras más resistentes en la cavidad bucal de estos pacientes es un factor de riesgo para desarrollar candidiasis invasivas de difícil manejo terapéutico.

La resistencia intrínseca de algunas especies de Candida especialmente a los azoles, junto con el desarrollo de resistencia debido a la profilaxis, ha obstaculizado el manejo de la candidiasis oral. Las pruebas de susceptibilidad antifúngica permiten una selección precisa del tratamiento y proporcionan contribuciones significativas para la comprensión de la epidemiología de la resistencia fúngica local y global ${ }^{(14)}$.

Al evaluar la frecuencia de sensibilidad de todas las $C$. no albicans frente al fluconazol, obtuvimos casi igual resultado que Terças et al. que informaron una sensibilidad del $60 \%$ para las cepas de $C$. no albicans ${ }^{(25)}$. Y un $13 \%$ de aislamientos con SDD, estos últimos correspondieron a $C$. glabrata. La baja sensibilidad de las $C$. no albicans al fluconazol en nuestro estudio fueron debidos a los aislamientos de $C$. krusei y C. glabrata, coincidente con el trabajo mencionado. C. glabrata se caracteriza por desarrollar resistencia al fluconazol, actualmente no cuenta con categoría sensible en la interpretación del CLSI y todos los aislamientos con CIM $\leq 32 \mathrm{ug} / \mathrm{ml}$ son considerados sensible dosis dependiente, por lo que se debe tener en cuenta que la terapia con este azol podría ser efectiva siempre que se utilicen altas dosis. Además, C. krusei es intrínsecamente resistente al fluconazol. Esto dificulta la selección de una terapia empírica durante el desarrollo de la candidiasis en los pacientes VIH positivos.

El voriconazol es generalmente activo frente a cepas resistentes al fluconazol como C. glabrata o C. krusei, aunque presenta frente a estas CIM mayores que para otras especies de Candida ${ }^{(36)}$. En este estudio los aislamientos de $C$. tropicalis, $C$. parapsilosis y C. krusei presentaron una sensibilidad del $100 \%$. Terças et al., informaron una sensibilidad del $75 \%$ frente a $C$. no albicans, sin embargo, esto fue debido a cepas resistentes de C. glabrata ${ }^{(25)}$. La sensibilidad de C. glabrata frente al voriconazol no fue determinada debido a que no posee puntos de corte establecidos por el CLSI actualmente. La resistencia a los azoles puede ser inducida por bombas de eflujo codificadas por los genes $M D R$ o $C D R$, por adquisición de mutaciones puntuales en el gen ERG11, que codifica para la enzima blanco de estos fármacos, o por la 
sustitución del ergosterol por otros esteroles en la membrana citoplasmática ${ }^{(37)}$. En el estudio de las C. no albicans solo se ensayaron dos antifúngicos, fluconazol y voriconazol.

Todas las cepas de $C$. albicans fueron sensibles frente a las equinocandinas, coincidiendo con lo informado por Pfaller et al. ${ }^{(38)}$. Estos antifúngicos presentan poco potencial de resistencia. La caspofungina ha demostrado ser eficaz en el tratamiento de la candidiasis orofaríngea y esofágica, en pacientes infectados por VIH, aunque se recomienda que se utilice solo en aquellos que presentan resistencia al fluconazol. La micafungina ha demostrado ser más eficaz que el fluconazol en la prevención de la candidiasis en pacientes inmunodeprimidos ${ }^{(33)}$.

En este estudio se obtuvo una gran sensibilidad de cepas de $C$. albicans frente a anfotericina B (98\%), similar a lo reportado por Grazziotin et al. y Nwezee et al. con $100 \%$ de sensibilidad $(4,41)$. Aunque el CLSI no ha establecido los puntos de corte interpretativos para la anfotericina $\mathrm{B}^{(10)}$, algunos investigadores han determinado que un valor $\leq 1 \mu \mathrm{g} / \mathrm{mL}$ correspondería a un aislado sensible a la anfotericina $B, y$ un valor superior sería resistente (39). Este antifúngico presenta un amplio espectro de acción, aunque se ha observado resistencia clínica y/o CIM elevadas, en algunas cepas como C. Iusitaniae, C. guilliermondii, C. lipolytica o C. tropicalis ${ }^{(40)}$.

El $97 \%$ de las cepas de C. albicans fueron sensibles a flucitosina. Solo el $3 \%$ mostró resistencia, coincidiendo con Blignaut et al. con $2,3 \%$ de resistencia ${ }^{(42)}$. Existen algunas cepas de Candida spp. que muestran resistencia primaria a la flucitosina, y cuando se utiliza en monoterapia, genera resistencia secundaria con facilidad $^{(32)}$.

Los resultados de esta investigación demuestran que los pacientes con VIH/SIDA son portadores de una amplia variedad de especies del género Candida, representando un factor de riesgo para el desarrollo de candidiasis orofaríngea en los mismos. La portación oral por cepas resistentes a los agentes antifúngicos más utilizados, especialmente a los azoles, podría representar un grave problema terapéutico en los individuos con VIH. Esto resalta la importancia de la identificación precisa y correcta de las especies de Candida, así como la necesidad de pruebas de susceptibilidad antifúngica previas a la implementación del tratamiento o profilaxis de la candidiasis oral en pacientes con VIH/SIDA.

Conflictos de interés: los autores declaran no tener conflictos de interés.

Financiación: Este estudio se realizó dentro del marco del Proyecto PINV15-919, financiado por el Consejo Nacional de Ciencia y Tecnología (CONACYT), Fondo para la Excelencia de la Educación y la Investigación (FEEI), Paraguay.

Contribución de autores: Todos los autores han contribuido en la elaboración del presente manuscrito.

\section{REFERENCIAS BIBLIOGRÁFICAS}

1. Oliva Ferrando MM, Bargagna $B$, Maldonado M, López. Evaluation of the prevalence of oral lesions in patients with HIV/AIDS and their identification: a systematic review. FASO. 2019; 26(1):72-7. Disponible en: http://faso.org.ar/revista/2019_26_1.ht $\underline{\mathrm{m}}$

2. Daniel HM, Lachance MA, Kurtzman CP. On the reclassification of species assigned to Candida and other anamorphic ascomycetous yeast genera based on phylogenetic circumscription. Antonie Van Leeuwenhoek. julio de 2014; 106(1):67-84. Doi: $10.1007 / \mathrm{s} 10482-014-0170-\mathrm{z}$
3. Das PP, Saikia L, Nath R, Phukan SK. Species distribution and antifungal susceptibility pattern of oropharyngeal Candida isolates from human immunodeficiency virus infected individuals. Indian J Med Res. 2016; 143(4):495-501. DOI: $10.4103 / 0971-$ 5916.184288

4. Nweze EI, Ogbonnaya UL. Oral Candida isolates among HIV-infected subjects in Nigeria. J Microbiol Immunol Infect. 2011; 44(3): 172-7. DOI: 10.1016/j.jmii.2011.01.028

5. Sullivan DJ, Westerneng TJ, Haynes KA, Bennett DE, Coleman DC. Candida dubliniensis sp. nov.: phenotypic and molecular characterization of a novel 
species associated with oral candidosis in HIV-infected individuals. Microbiology. 1995; 141(7): 1507-21. DOI: $10.1099 / 13500872-141-7-1507$

6. Ahmad S, Khan Z, Asadzadeh M, Theyyathel A, Chandy R. Performance comparison of phenotypic and molecular methods for detection and differentiation of Candida albicans and Candida dubliniensis. BMC Infect Dis. 2012; 12: 230. DOI: 10.1186/14712334-12-230

7. Arendrup MC, Patterson TF. MultidrugResistant Candida: Epidemiology, Molecular Mechanisms, and Treatment. J Infect Dis. 2017; 216(suppl_3): S44551. DOI: $10.1093 /$ infdis/jix131

8. Sanabria $R$, Samudio $M$, Fariña $N$, Laspina F, Canese $\mathrm{JO}$ de, Ledesma GA, et al. Identification of candida species isolated from ambulatory, hospitalized and immune-depressed patients in Paraguay. Mem Inst Investig En Cienc Salud. 2006;4(2):45-8. Disponible en: http://scielo.iics.una.py/scielo.php?scrip $\mathrm{t}=\mathrm{sci}$ arttext\&pid=S181295282006000200008\&lng=en.

9. Clinical and Laboratory Standards Institute (CLSI). Performance standards for antifungal susceptibility testing of yeasts. M 60. Wayne, PA: CLSI, 2017.

10. Clinical and Laboratory Standards Institute (CLSI). Method for antifungal disk diffusion susceptibility testing of yeast, $2^{\text {nd }}$ ed. CLSI Document M44-A2. Wayne, PA: CLSI, 2009

11. Li $X$, Lei $L$, Tan D. Oropharyngeal Candida colonization in human immunodeficiency virus infected patients. 121. 2012; 5: 375-402. DOI: $10.1111 / \mathrm{apm} .12006$

12. Araiza J, Magallón-Zazueta $\mathrm{L}$, Contreras-García S, Tirado-Sánchez A, Bonifaz A. Oral candidiasis in patients with HIV / AIDS; clinical and etiological spectrum. Rev Médica MD. 2018;9.10(4):322-7. Disponible en: https://www.medigraphic.com/cgibin/new/resumen.cgi?IDARTICULO $=826$ $\underline{26}$

13. Castrillo $S$, Castrillo $M$, Alvarez $M$, Castrillo N. Candida species in HIV / AIDS patients with Oropharyngeal Candidiasis. Acta Odontológica Venez. 2012; 50(3). Disponible en: https://www.actaodontologica.com/edic iones/2012/3/art-5/

14. Goulart LS, Souza WWR de, Vieira CA, Lima JS de, Olinda RA de, Araújo C de, et al. Oral colonization by Candida species in HIV-positive patients: association and antifungal susceptibility study. Einstein São Paulo. 2018;16(3). DOI: $10.1590 / \mathrm{S} 1679-45082018 \mathrm{AO} 4224$
15. Castro-Alegría LÁ, Álvarez-Valle MI, Martínez-Buitrago E. Candida in the oral cavity of HIV patients in Cali, Colombia: determination of species and sensitivity to fluconazole. Iatreia. 2015; 28(4): 368-77. Doi: 10.17533/udea.iatreia.v28n4a02

16. Clark-Ordóñez I, Callejas-Negrete $O A$, Aréchiga-Carvajal ET, Mouriño-Pérez RR. Candida species diversity and antifungal susceptibility patterns in oral samples of HIV/AIDS patients in Baja California, Mexico. Med Mycol. 2017; 55 (3): 285-94.

Doi:

\section{$10.1093 / \mathrm{mmy} / \mathrm{myw} 069$}

17. Yorio VP. Candida dubliniensis and Candida albicans: phenotypically and phylogenetically related opportunistic oral pathogens. Actas Odontológicas Publ Discontinuada. 2006; 3(1):36-41. DOI: $10.22235 / a 0 . v 3 i 1.988$

18. Abharian $\mathrm{PH}$, Dehghan $\mathrm{P}, \mathrm{Abharian} \mathrm{PH}$, Tolouei S. Molecular characterization of Candida dubliniensis and Candida albicans in the oral cavity of drug abusers using duplex polymerase chain reaction. Curr Med Mycol. 2018; 4(1):12. Doi: $10.18502 / \mathrm{cmm} \cdot 4.1 .29$

19. Luque AG, Biasoli MS, Tosello ME, Binolfi A, Lupo S, Magaró HM. Oral yeast carriage in HIV-infected and noninfected populations in Rosario, Argentina. Mycoses. 2009; 52 (1): 539. Doi: 10.1111/j.14390507.2008.01542.x

20. Satana D, Gonca E, Genc Z, Erturan Z. The antifungal susceptibilities of oral Candida spp. isolates from HIV-infected patients. Afr J Microbiol Res. 2010; 4(6):466-70. Disponible en: https://academicjournals.org/journal/AJ MR/article-abstract/C203B6612087

21. Chavasco JK, Paula CR, Hirata $\mathrm{MH}$, Aleva NA, Melo CE de, Gambale W, et al. Molecular identification of Candida dubliniensis isolated from oral lesions of HIV-positive and HIV-negative patients in São Paulo, Brazil. Rev Inst Med Trop São Paulo. 2006; 48(1):21-6. DOI: 10.1590/S0036-46652006000100005

22. Mariel-Carballo G. Phenotypic identification of Candida dubliniensis isolated from oral mucosa candidiasis in immunosuppressed patients. Dermatol Rev Mex. 2019;63(1):14-25. Disponible en: https://www.medigraphic.com/cgi$\underline{\mathrm{bin} / \mathrm{new} / \text { resumenI.cgi?IDARTICULO }=85}$ 014

23. Llanos González I, Montoya Ojeda R, Puello Hoyos M, Young Castro G, Correa Jiménez O, Suárez Álvarez P. Carriage of Candida spp. in the oral cavity in diabetics and non-diabetics. Rev Cuba 
Endocrinol. 2017; 28(3): 1-11. Disponible en:

http://scielo.sld.cu/scielo.php?script $=$ sc i abstract\&pid=S1561-

$29532017000300007 \& \operatorname{lng}=$ es\&nrm $=$ iso

\&tlng=es

24. Kirkpatrick WR, Revankar SG, Mcatee RK, Lopez-Ribot JL, Fothergill AW, McCarthy DI, et al. Detection of Candida dubliniensis in oropharyngeal samples from human immunodeficiency virusinfected patients in North America by primary CHROMagar candida screening and susceptibility testing of isolates. J Clin Microbiol. 1998; 36(10): 3007-12. Doi: 10.1128/JCM.36.10.30073012.1998

25. Terças ALG, Marques SG, Moffa EB, Alves MB, de Azevedo CMPS, Siqueira $W L$, et al. Antifungal Drug Susceptibility of Candida Species Isolated from HIVPositive Patients Recruited at a Public Hospital in São Luís, Maranhão, Brazil. Front Microbiol. 2017; 8: 298. DOI: 10.3389/fmicb.2017.00298

26. Zuluaga A, Arango-Bustamante $\mathrm{K}$, Caceres DH, Sánchez-Quitian ZA, Velásquez $\mathrm{V}$, Gómez $\mathrm{BL}$, et al. Concordance analysis between different methodologies used for identification of oral isolates of Candida species. Colomb Médica. 2018; 49 (3): 193-200. DOI: 10.25100/cm.v49i3.3774

27. Ochiuzzi ME, Cataldi S, Guelfand L, Maldonado I, Arechavala A. Evaluation of the Vitek 2 system for the identification of the main yeast species of the Candida genus. Rev Argent Microbiol. 2014; 46(2): 107-10. DOI: 10.1016/S0325-7541(14)70058-6

28. Garzón C, Gutiérrez L, Salazar C, Barrientos S, Romero C. Candida's species in relation with CD4 counts in HIV-AIDS patients. 27. 2008; 59: 2933. Disponible

en: https://dialnet.unirioja.es/servlet/articul o?codigo $=3986456$

29. Rao RP. Isolation, identification and speciation of Candida species from various clinical samples in a tertiary care teaching Hospital in Karnataka, India. J Evid Based Med Healthc. 2019; 6(11):866-8. DOI:

10.18410/jebmh/2019/182

30. Gutiérrez $C$, de Bedout $C$, Tobón $A$, Cano L, Arango M, Tabares A, et al. Sensitivity to Fluconazol and Voriconazol in Candida spp. isolates from the oral mucosa of HIV positive patients. Infectio. 2007; 11(4):183-9. Disponible en:

http://www.scielo.org.co/scielo.php?scri pt=sci_abstract\&pid $=$ S0123-
$93922007000400005 \&$ Ing $=$ en \&nrm $=$ iso \&tlng=es

31. Ortega Riveros M. Estudio de la patogenicidad de Candida y de la actividad de los fármacos antifúngicos en un modelo animal en Caenorhabditis elegans [Internet] [Tesis Doctoral]. Universidad del País Vasco - Euskal Herriko Unibertsitatea; 2015 [citado 27 de marzo de 2020]. Disponible en: https://dialnet.unirioja.es/servlet/tesis? codigo $=100132$

32. Brito D, Fernández J, Castillo $M$, Azuero $S$, Hernández $R$, Saúl $Y$, et al. Fluconazole and voriconazole susceptibility in oral colonization isolates of Candida spp. in HIV patients. Invest Clin. 2019; 60(4):275-82. Disponible en: https://produccioncientificaluz.org/inde x.php/investigacion/article/view/31668

33. Khedri $S$, Santos ALS, Roudbary $M$, Hadighi $R$, Falahati $M$, Farahyar $S$, Khoshmirsafa $M$, Kalantari S. Iranian HIV/AIDS patients with oropharyngeal candidiasis: identification, prevalence and antifungal susceptibility of Candida species. Lett Appl Microbiol. 2018 Oct; 67(4): 392-9. doi: 10.1111/lam.13052. Epub 2018 Aug 16. PMID: 30019443.

34. Aguilar G, Araujo $P$, Lird G, Insaurralde S, Kawabata A, Ayala E, et al. Identificación y perfil de sensibilidad de Candida spp. aisladas de hemocultivos en hospitales de Paraguay. Rev Panam Salud Publica. 2020; 44: e34. https://doi.org/10.26633/RPSP.2020.34

35. Ochiuzzi ME, Arechavala A, Guelfand L, Maldonado I, Soloaga R. Evaluation of the VITEK 2 system (AST-YSO1 cards) for antifungal susceptibility testing against different Candida species. Rev Argent Microbiol. 2014; 46(2):1118.

36. Estrella MC. Antifungals in the treatment of systemic infections: importance of the mechanism of action, spectrum of activity and resistance. Rev Esp Quimioter. 2010; 23(4):169-76. Disponible

en: https://dialnet.unirioja.es/servlet/articul o?codigo $=3705373$

37. Valencia Torres B. Antifungal resistance mechanisms and new develping antifungals [Internet] [Trabajo de Grado]. Universidad Complutense; 2018. Disponible en: http://147.96.70.122/Web/TFG/TFG/Me moria/BEATRIZ\%20VALENCIA\%20TORR ES.pdf

38. Pfaller M, Boyken L, Hollis R, Kroeger J, Messer S, Tendolkar S, et al. In vitro susceptibility of invasive isolates of 
Candida spp. to anidulafungin, caspofungin, and micafungin: six years of global surveillance. J Clin Microbiol. 2008; 46: 150-6. DOI:

10.1128/JCM.01901-07

39. Kaur R, Dhakad MS, Goyal R, Haque A, Mukhopadhyay G. Identification and Antifungal Susceptibility Testing of Candida Species: A Comparison of Vitek-2 System with Conventional and Molecular Methods. J Glob Infect Dis. 2016;8(4):139-46. DOI: 10.4103/0974777X.192969

40. Catalán M, Montejo JC. Systemic antifungals. Pharmacodynamics and pharmacokinetics. Rev Iberoam Micol. 2006; 23(1): 39-49. DOI: 10.1016/S1130-1406(06)70012-2
41. Grazziotin NA, Danielli LJ, Maroso CT, Madalozzo VA, Jaskulski $M$ da $R$. Susceptibility of Candida isolates from HIV-positive patients to azoles and amphotericin B. Saúde St Maria. 2015; 41(2): $169-74$.

DOI: $10.5902 / 2236583417006$

42. Blignaut E, Messer S, Hollis RJ, Pfaller MA. Antifungal susceptibility of South African oral yeast isolates from HIV/AIDS patients and healthy individuals. Diagn Microbiol Infect Dis. 2002; 44(2):169-74.

DOI: 10.1016/S0732-8893(02)00440-6. 